\title{
Oxidative stress is increased in serum from Mexican patients with relapsing-remitting multiple sclerosis
}

\author{
Genaro Gabriel Ortiz ${ }^{\mathrm{a}, \mathrm{b}, *}$, Miguel Ángel Macías-Islas ${ }^{\mathrm{d}}$, Fermín P. Pacheco-Moisése, \\ José A Cruz-Ramos ${ }^{\mathrm{a}}$, Silvia Sustersik ${ }^{\mathrm{a}}$, Elías Alejandro Barba ${ }^{\mathrm{b}}$ and Adriana Aguayo ${ }^{\mathrm{f}}$ \\ ${ }^{a}$ Laboratorio de Estrés Oxidativo \& Patología, División de Neurociencias, Centro de Investigación Biomédica de \\ Occidente (CIBO), Instituto Mexicano del Seguro Social (IMSS), Guadalajara, Jalisco, México \\ ${ }^{\mathrm{b}}$ División de Ciencias de la Salud, Instituto Tecnológico de Estudios Superiores de Monterrey (ITESM) Campus \\ Guadalajara, Zapopan, Jalisco, México \\ ${ }^{\mathrm{c}}$ Departamento de Neurología, Unidad Médica de Alta Especialidad (UMAE)- Hospital de Especialidades, Centro \\ Médico Nacional de Occidente (CMNO), IMSS, Guadalajara, Jalisco, México \\ ${ }^{\mathrm{d}}$ Departamento de Neurosciences, CUCS, Universidad de Guadalajara, Guadalajara, Jalisco, México \\ e Departamento de Química, Centro Universitario de Ciencias Exactas e Ingenierías, Universidad de Guadalajara, \\ Guadalajara, Jalisco, México \\ ${ }^{\mathrm{f}}$ Fundación Mexicana para la Esclerosis Múltiple A.C. Guadalajara, Jalisco, México
}

\begin{abstract}
Objective: To determine the oxidative stress markers in serum from patients with relapsing-remitting multiple sclerosis. Methods: Blood samples from healthy controls and 22 patients 15 women (7 aged from 20 to 30 and 8 were $>40$ years old) and 7 men (5 aged from 20 to 30 and 2 were $>40$ years old) fulfilling the McDonald Criteria and classified as having Relapsing-Remitting Multiple Sclerosis accordingly with Lublin were collected for oxidative stress markers quantification.

Results: Nitric oxide metabolites (nitrates/nitrites), lipid peroxidation products (malondialdehyde plus 4-hidroxialkenals), and glutathione peroxidase activity were significantly increased in serum of subjects with relapsing-remitting multiple sclerosis in comparison with that of healthy controls. These data support the hypothesis that multiple sclerosis is a component closely linked to oxidative stress.
\end{abstract}

Keywords: Multiple sclerosis, oxidative stress, nitric oxide, lipid peroxidation, glutathione peroxidase

\section{Introduction}

Multiple sclerosis (MS) is one of the most common chronic and disabling disorders of the central nervous system (CNS) in young adults. Little is known about its etiology, although evidence indicates that MS is a complex trait caused by interactions of genetic and en-

\footnotetext{
* Corresponding author: Genaro G. Ortiz MD., PhD, Laboratorio Estrés Oxidativo \& Patología, CIBO-IMSS. \& ITESM, Sierra Mojada 800 C.P. 44340, Guadalajara, Jalisco, México. E-mail: genarogabriel@yahoo.com.
}

vironmental factors. Several patterns of MS remissionexacerbation have been identified and described; typically MS begins as a relapsing illness with episodes of neurological dysfunction lasting several weeks, followed by partial or complete improvement (relapsing remitting MS). At a later stage insidious accumulation of disability is obtained [29].

There is increasing evidence that multiple sclerosis is not only characterized by immune mediated inflammatory reactions but also by neurodegenerative processes [10]. Bursts of focal inflammation are thought to underlie the episodic, relapsing - remitting phase 
of MS, whereas axonal loss and neurodegeneration are responsible for progressive symptoms, which are the predominant cause of disability [11].

In neurodegenerative diseases, neuronal loss is mediated by oxidative stress which constitutes a final common toxic pathway. It has been hypothesized that relapsing-remitting phase of MS is mediated by oxidative stress [9]. Some oxidative stress markers such as: plasma cholesteryl ester hidroperoxides [6]; nitrate and nitrite in serum [8] and blood leukocytes [14]; diene conjugates in peripheral blood leukocytes are all higher in patients with MS than the healthy control subjects. In addition, a significant decrease in the level of the natural scavenger of peroxynitrite, uric acid $[19,25]$ has been described in MS patients. On the other hand, the glutathione peroxidase activity in MS remains unclear, for example it has been reported a decreased enzymatic activity in blood cells $[13,16,26]$, increased activity [30], or a normal activity in lymphocytes, granulocytes and platelets of MS patients [27]. The contradictory reported data for glutathione peroxidase activity prompt us to investigate this activity and oxidative stress markers (nitric oxide metabolites and lipid peroxidation products) in serum of Mexican patients with relapsing-remitting multiple sclerosis.

\section{Material and methods}

\subsection{Patients}

The prevalence of MS varies significantly depending on the genetic background of the patient [3]. MS is considered a low prevalence of disease in Mexico, its features have been described in studies of several geographical regions of the country, remarkably the clinical profile of the disease is similar to that reported in other countries and the pattern observed corresponded to that found in northern latitudes [4,28]. Blood samples were obtained from 22 patients who attended the consulting room at the hospital (Unidad Médica de Alta Especialidad - Hospital de Especialidades, Centro Médico Nacional de Occidente del Instituto Mexicano del Seguro Social). None of all the patients were experiencing an acute relapse at the time of blood sampling. Informed consent was obtained from the patient by a physician staff. The study was approved by the UMAE-HE reviewed board and was carried out in accordance with the principles of declaration of Helsinki as revised 2000. The diagnosis of MS was made following the revised McDonald Criteria $2005[17,20]$ and patients were classified as having Relapsing-Remitting Multiple Sclerosis accordingly with Lublin [15]. Patients and volunteers were matched for sex and age. In MS group, there were 15 women ( 7 aged from 20 to 30 and 8 were $>40$ years old) and 7 men ( 5 aged from 20 to 30 and 2 were $>40$ years old). The mean age of the MS group was 32 years. None of the selected patients and control subjects was taking antioxidants.

Fasting blood samples from patients and control subjects were obtained between 9:00 and 11:00 AM. Blood was drawn from a vein in the antecubital fossa by a 19gauge needle. The tourniquet was carefully released after its application and blood was collected. Serum was obtained by a standard technique. Protein concentration was determined by Bradford method using bovine serum albumin as a standard [1].

\subsection{Nitric oxide}

Nitric oxide release was determined spectrophotometrically by measuring the accumulation of its stable degradation products, nitrite and nitrate. Quantification of these metabolites in serum was done using a commercial package (Calbiochem Nitric Oxide Assay Kit, colorimetric 482650). Briefly, Nitrate to nitrite conversion was done using nitrate reductase. Total nitrite is then determined spectrophotometrically by using the Griess reaction. Results are expressed as $\mu \mathrm{M} / \mathrm{mL}$.

\subsection{Lipid peroxidation}

Serums were tested for lipid peroxidation using a Kit from Oxford Biomedical Res Inc. (product No FR 12). Each measurement was repeated four times. The Kit contains a chromogenic reagent (Nmethyl-2-phenylindole) which reacts with the lipid peroxidation products malonaldehyde (MDA) and 4Hydroxyalkenals (4-OHA) at $45^{\circ} \mathrm{C}$ yielding a stable chromophore with maximal absorbance at a wavelength of $586 \mathrm{~nm}$ [4]. Results are expressed as nmol MDA+4OHA (mg protein $)^{-1}$.

\subsection{Glutathione peroxidase activity}

Glutathione peroxidase activity was determined according to the method described previously [7] which is based on the oxidation of reduced glutathione (GSH) in the presence of cumene, glutathione reductase and NADPH. Enzymatic activity was referred as $\mu \mathrm{M}$ of oxidized NADPH $\min ^{-1}$ (mg of protein $)^{-1}$. 


\subsection{Statistical analysis}

Data are expressed as mean values \pm SEM. Differences in oxidative stress markers were assessed with the nonparametric Wilcoxon signed rank test for paired data. A $p$ value $<0.05$ was considered statistically significant.

\section{Results and discussion}

Free radicals (nitric oxide, superoxide radical, etc.) production leads to oxidative stress when there is an imbalance in the redox status of a cell [23]. Free radicals are generally short lived and thus, indirect methods of detection are required. Standard detection methods include: electron spin resonance, thiobarbituric acid reactive substances, detection of malonaldehyde, and other oxidation products from polyunsaturated fatty acids (such as 4hydroxynonenal), measurement of oxidation products such as isoprostanes, 8-hydroxydeoxyguanosine, methionine sulfoxide, etc. [21]. In this work we measured lipid oxidation products (malondialdehyde plus 4-hidroxialkenals) and oxide nitric metabolites (nitrates/nitrites) as a measure of oxidative injury. Malondialdehyde and 4-hidroxialkenals are abundant diffusable products thought to contribute significantly to the deleterious effects of lipid peroxidation in tissues. Reactive aldehydes from lipid peroxidation adduct a number of cellular nucleophiles, including proteins, nucleic acids, and some lipids [5]. On the other hand, nitric oxide has a primary play a role in pathophysiology of MS. In active MS lesions, microglia and activated astrocytes express the enzyme inducible nitric oxide synthase which enables them to produce large amounts of nitric oxide [2].

Figure 1 shows serum levels of metabolites of nitric oxide (A), lipid peroxidation products (B) in healthy matched subjects and patients with relapsing-remitting multiple sclerosis. In serum samples from patients these values are significantly higher than in healthy controls (A-B). The increases in lipid peroxidation products and nitric oxide metabolites were 1.6 and 2.3 times, respectively in comparison with samples from control subjects; it shows a high oxidative stress in serum from MS patients. On the other hand, the activity of glutathione peroxidase was significantly increased (1.3 times) in serum of MS patients with that of healthy controls (Fig. 1C).

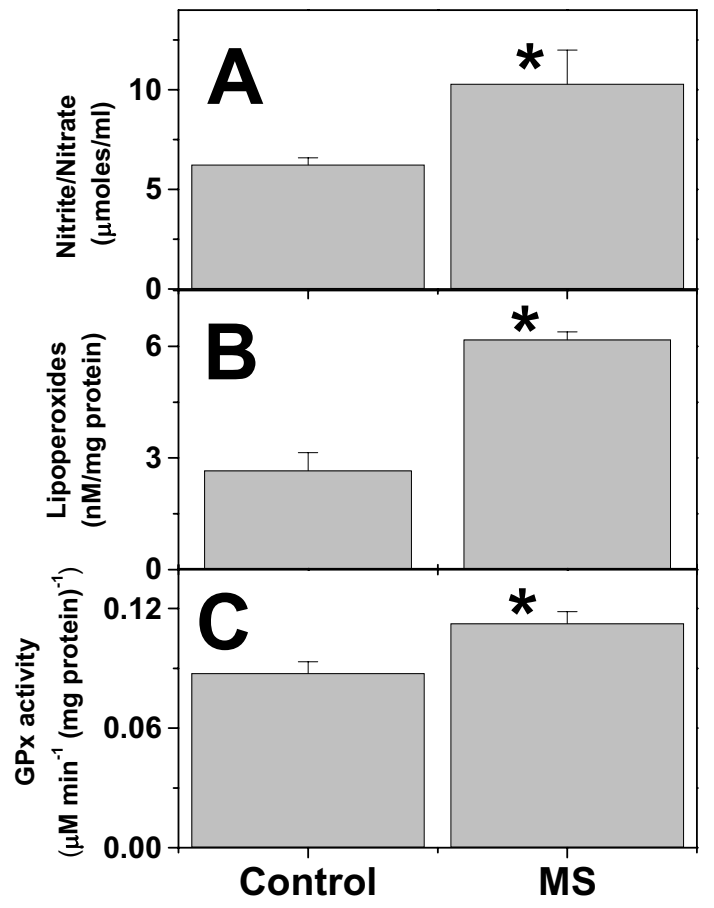

Fig. 1. Nitric oxide catabolites (A), lipid peroxidation products (B) and glutathione peroxidise activity $(\mathrm{C})$ in serum from healthy controls and patients with relapsing-remitting multiple sclerosis. Asterisks indicate a significant difference from the multiple sclerosis patients $(\mathrm{P}<0.05)$. The bar indicates the mean \pm S.E.

Lower serum uric acid level in MS patients has been reported [19]. The higher levels of oxide nitric metabolites found in this work could be relevant in MS development if there is a low concentration of serum uric acid. Recent evidence suggests that nitric oxide contributes to the disease process of MS by blood brain barrier breakdown and by direct tissue damage, blocking axonal conduction and by inducing axonal degeneration and plaque formation [12,24]. In addition, peroxynitrite (the reaction product of nitric oxide and superoxide anion) mediate several potentially destructive chemical reactions, including tyrosine nitration and lipid peroxidation and its effects are consistently associated with active lesions and during the remitting phase. Peroxynitrite participates to neuron and oligodendrocyte damage in association with inflammatory processes [9]. In addition, lipid peroxidation can trigger the process of apoptosis, activating the intrinsic suicide pathway present within all cells [18].

The role of glutathione peroxidase in peripheral tissues of MS patients remains unclear since the contradictory data regarding its activity $[13,16]$. Glutathione peroxidase is a free-radical scavenger enzyme, therefore is an important part of the antioxidant cellular 
defense system. The increase in its activity found in serum of MS subjects could be a response to oxidative stress in order to minimize cell injury. Studies are in progress, in our laboratory, to strengthen these observations and to investigate the role of mitochondrial enzymatic activities in the reactive species production in MS.

The etiology of MS has not yet been fully elucidated; however it is believed that the inflammatory environment in demyelinating lesions lead to the generation of oxygen and nitrogen free radicals as well as proinflammatory cytokines which contribute to the development and progression of the disease [22,24]. The production of the reactive oxygen and nitrogen species is an inherent property of activated immune cells. Additionally, reactive oxygen species are involved in phagocytosis and degradation of myelin, oligodendrocyte damage, and axonal degeneration [23]. Inflammation can lead to oxidative stress and conversely. Thus, oxidative stress and inflammation are involved in a self-perpetuating cycle.

The novel information provided by this work is that oxidative stress is increased in serum from MS patients even when they do not have a relapse in the disease. Whether or not this phenomenon reflects a similar process occurring in the central nervous system of MS subjects could be a matter of controversy. However, the existence of this phenomenon can not be minimized, since oxidative stress is a major pathway of cell damage.

\section{Acknowledgments}

This work was supported by Tevas Neurosciences México.

\section{References}

[1] M. Bradford, A rapid and sensitive method for the quantification of microgram quantities of proteins utilizing the principle of protein-dye binding, Analyt Biochem 72 (1976), 248-256.

[2] O. Bagasra, F.H. Michaels, Y.M. Zheng et al., Activation of the inducible form of nitric oxide synthase in the brains of patients with multiple sclerosis, Proc Natl Acad Sci U S A 92 (1995), 12041-12045.

[3] A. Compston, Genetic epidemiology of multiple sclerosis, $J$ Neurol Neurosurg Psychiatry 62 (1997), 553-561.

[4] J. Cordova, S. Vargas and J. Sotelo, Western and Asian features of multiple sclerosis in Mexican Mestizos, Clin Neurol Neurosurg 109 (2007), 146-151.

[5] H. Esterbauer and K.H. Cheeseman, Determination of aldehydic lipid peroxidation products: malonadehyde and 4hidroxynonenal, Meth Enzymol 186 (1990), 407-421.
[6] G. Ferretti, T. Bacchetti, F. Principi el al., Increased levels of lipid hydroperoxides in plasma of patiens with multiple sclerosis: a relationship with paraoxonase activity, Multiple Sclerosis 11, (2005), 677-682.

[7] L. Flohe and W.A. Gunzler, Assay of glutathione peroxidase, Meth enzymol 105 (1984), 114-121.

[8] G. Giovannoni, S.J.R. Heales, N.C. Silver et al., Raised serum nitrate and nitrite levels in patients with multiple sclerosis, $J$ Neurol Sci 145 (1997), 77-81.

[9] R.E. Gonsette, Oxidative stress and excitotoxicity: a therapeutic issue in multiple sclerosis? Multiple Sclerosis 14 (2008), 22-34.

[10] S.L. Hauser and J.R. Oksenberg, The neurobiology of multiple sclerosis: genes, inflammation, and neurodegeneration, Neuron 52 (2006), 61-76.

[11] B. Hemmer, J.J. Archelos and H-P. Hartung, New concepts in the immunopathogenesis of multiple sclerosis, $\mathrm{Nat} \mathrm{Rev} \mathrm{Neu-}$ rosci 3 (2002), 291-301.

[12] K.E. Hill, L.V. Zollinger, H.E. Watt et al., Inducible nitric oxide synthase in chronic active multiple sclerosis plaques: distribution, cellular expression and association with myelin damage, J Neuroimmunol 151 (2004), 171-179.

[13] G.E. Jensen, G. Gissel-Nielsen and J.J. Clausen, Leucocyte glutathione peroxidase activity and selenium level in multiple sclerosis, Neurol Sci 48 (1980), 61-67.

[14] M. Koch, J. Mostert, A. Arutjunyan et al., Peripheral blood leukocyte NO production and oxidative stress in multiple sclerosis, Multiple Sclerosis 14 (2008), 159-165.

[15] F.D. Lublin and S.C. Reingold, Defining the clinical course of multiple sclerosis: results of an international survey. National Multiple Sclerosis Society (USA) Advisory Committee on Clinical Trials of New Agents in Multiple Sclerosis, Neurology 46 (1996), 907-911.

[16] G.L. Mazzellaa, E. Sinforiania, F. Savoldia et al., Blood Cells Glutathione Peroxidase Activity and Selenium in Multiple Sclerosis, Eur Neurol 22 (1983), 442-446.

[17] W.I. McDonald, A. Compston, G. Edan et al., Recommended diagnostic criteria for multiple sclerosis: guidelines from the international panel on the diagnosis of multiple sclerosis, Ann Neurol 50 (2001), 121-127.

[18] J.H. Noseworthy, C. Lucchinetti, M. Rodriguez et al., Multiple sclerosis, N Engl J Med 343 (2000), 938-952.

[19] F. Peng, B. Zhang, X. Zhong et al., Serum uric acid levels of patients with multiple sclerosis and other neurological diseases, Multiple Sclerosis 14 (2008), 188-196.

[20] C.H. Polman, S.C. Reingold, G. Edan et al., Diagnostic criteria for multiple sclerosis: 2005 revisions to the "McDonald Criteria”, Ann Neurol 58 (2005), 840-846.

[21] W.A. Pryor, On the detection of lipid hydroperoxides in biological samples, Free Radic Biol Med 7 (1989), 177-178.

[22] G. Schreibelt, J. van Horssen, S. van Rossum et al., Therapeutic potential and biological role of endogenous antioxidant enzymes in multiple sclerosis pathology, Brain Res Rev 56 (2007), 322-330.

[23] K.J. Smith, R. Kapoor and P.A. Felts, Demyelination: the role of reactive oxygen and nitrogen species, Brain Pathol 9 (1999), 69-92.

[24] K. Smith and H. Lassmann, The role of nitric oxide in multiple sclerosis, Lancet Neurol 1 (2002), 232-241.

[25] S. Sotgiu, M. Pugliatti, A. Sanna et al., Serum uric acid and multiple sclerosis, Neurol Sci 23 (2002), 183-188.

[26] A. Szeinberg, R. Golan, J. Ben Ezzer et al., Decreased erythrocyte glutathione peroxidase activity in multiple sclerosis, Acta Neurol Scand 60 (1979), 265-221. 
[27] A. Szeinberg, R. Golan, J. Ben-Ezzer et al., Glutathione peroxidase activity in various types of blood cells in multiple sclerosis, Acta Neurol Scand 63 (1981), 67-75.

[28] M. Velázquez-Quintana, M.A. Macías-Islas, V. Rivera-Olmos et al., [Multiple sclerosis in Mexico: a multicentre study], Rev Neurol 36 (2003), 1019-1022.

[29] B.G. Weinshenker, B. Bass, G.P. Rice, J. Noseworthy et al.,
The natural history of multiple sclerosis: a geographically based study. I. Clinical course and disability, Brain 112 (1989), 133-146.

[30] B. Zachara, J. Gromadzinacuteska, J. Czernicki et al., Red blood cell glutathione peroxidase activity in multiple sclerosis, J Mol Med 62 (1984), 179-182. 


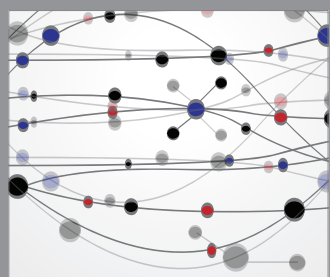

The Scientific World Journal
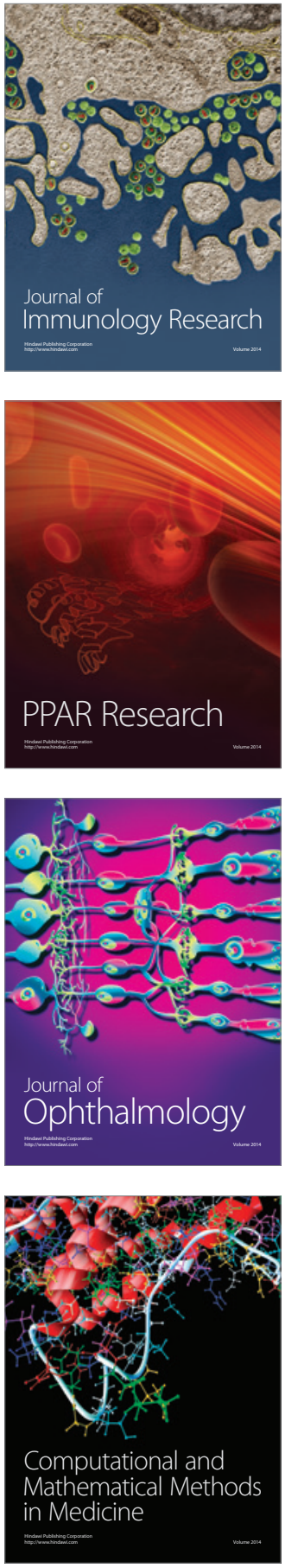

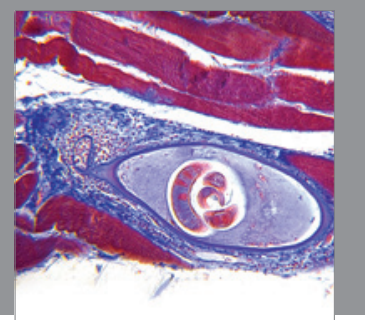

Gastroenterology

Research and Practice
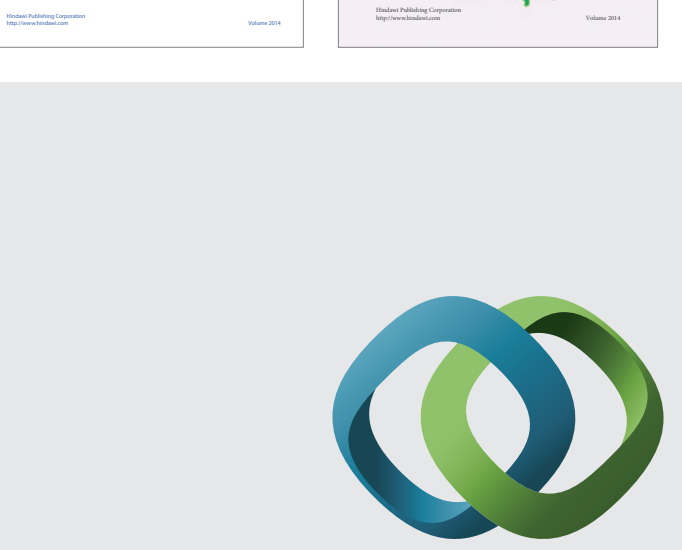

\section{Hindawi}

Submit your manuscripts at

http://www.hindawi.com
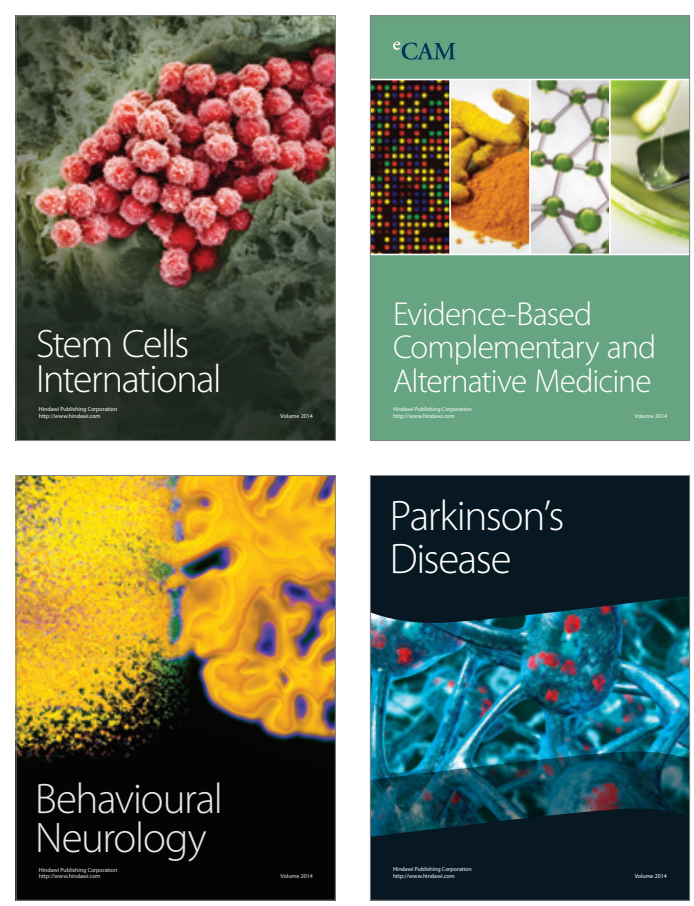

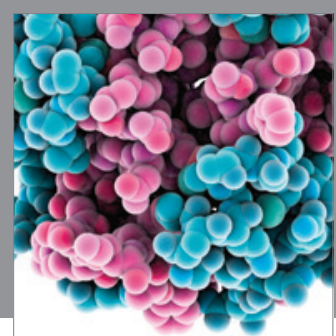

Journal of
Diabetes Research

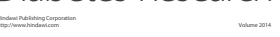

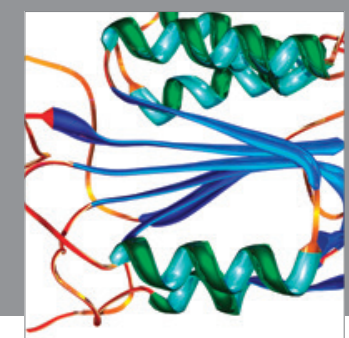

Disease Markers
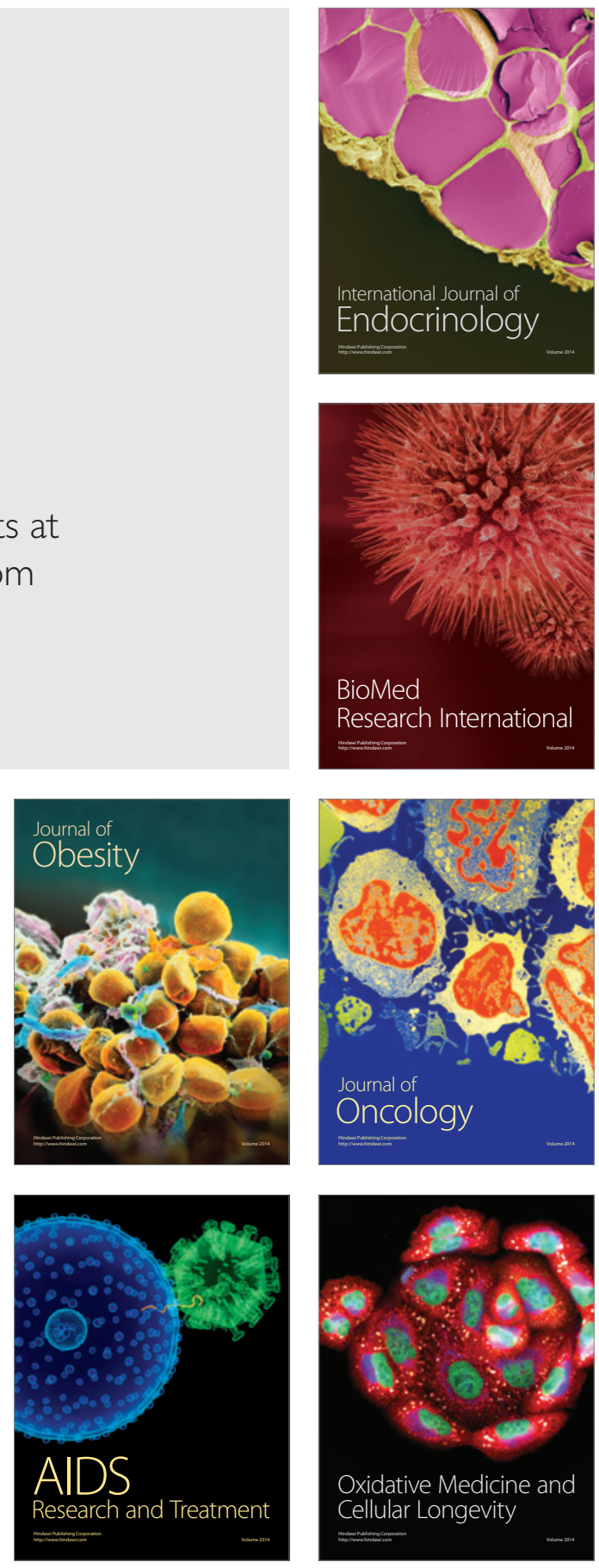